\title{
Toward Live Streamed Improvisational Game Experiences
}

\author{
Chance Lytle \\ Carnegie Mellon University \\ Entertainment Technology Center \\ Pittsburgh, Pennsylvania \\ cllytle@andrew.cmu.edu \\ Trace Dressen \\ Carnegie Mellon University \\ Entertainment Technology Center \\ Pittsburgh, Pennsylvania \\ tdressen@andrew.cmu.edu
}

\author{
Parker Ramsey \\ Carnegie Mellon University \\ Entertainment Technology Center \\ Pittsburgh, Pennsylvania \\ cramsey@andrew.cmu.edu \\ Dong hyun Kang \\ Carnegie Mellon University \\ Entertainment Technology Center \\ Pittsburgh, Pennsylvania \\ dkang2@andrew.cmu.edu
}

\author{
Joey Yeo \\ Carnegie Mellon University \\ Entertainment Technology Center \\ Pittsburgh, Pennsylvania \\ joeyy@andrew.cmu.edu \\ Brenda Bakker Harger \\ Jessica Hammer \\ Carnegie Mellon University \\ Pittsburgh, Pennsylvania \\ bharger@andrew.cmu.edu \\ hammerj@andrew.cmu.edu
}

\begin{abstract}
In this project, we explore the intersection of improvisational theater and Twitch live streaming. Improv offers social affordances to live streams, while streaming offers improv performers a way to both broaden their reach to non-copresent audiences and create new types of participatory improv games. Based on insights from both domains, we present StAGE, a live streaming system that both communicates performative information otherwise lost when streaming live theater and accommodates participatory roles for performers, hosts, and audiences. Using our system, we created and playtested four improv game prototypes exploring a range of interactions. We show that StAGE affords novel interactions both as a streaming experience and as improvisational theater. We propose that the social transfer of roles and information, particularly by hosts, is a critical aspect of the design. We conclude by discussing newly discovered challenges and potential for the future of the intersection of these domains.
\end{abstract}

\section{CCS CONCEPTS}

- Human-centered computing $\rightarrow$ Interaction paradigms; Interface design prototyping; • Applied computing $\rightarrow$ Computer games.

\section{KEYWORDS}

livestreaming; audience participation; improv, improvisational theater

\section{ACM Reference Format:}

Chance Lytle, Parker Ramsey, Joey Yeo, Trace Dressen, Dong hyun Kang, Brenda Bakker Harger, and Jessica Hammer. 2020. Toward Live Streamed Improvisational Game Experiences. In Proceedings of the Annual Symposium on Computer-Human Interaction in Play (CHI PLAY '20), November 2-4,

Permission to make digital or hard copies of part or all of this work for personal or classroom use is granted without fee provided that copies are not made or distributed for profit or commercial advantage and that copies bear this notice and the full citation on the first page. Copyrights for third-party components of this work must be honored.

For all other uses, contact the owner/author(s).

CHI PLAY '20, November 2-4, 2020, Virtual Event, Canada

(C) 2020 Copyright held by the owner/author(s).

ACM ISBN 978-1-4503-8074-4/20/11.

https://doi.org/10.1145/3410404.3414226
2020, Virtual Event, Canada. ACM, New York, NY, USA, 12 pages. https: //doi.org/10.1145/3410404.3414226

\section{INTRODUCTION}

Livestreaming platforms such as Twitch are increasingly exploring the value of audience participatory experiences, from Creative channels where audiences can get involved in streamer creative production [52, 54] to audience participation games [47]. Preliminary research on audience participation in streaming environments has identified design challenges such as attention management, player agency, and latency [16]. Creating novel experiences for livestreamed audience participation means addressing these challenges.

To better understand these design challenges, we look to improvisational theater, in which performers integrate audience suggestions into a live theatrical performance. Improvisers have deep expertise in co-creating live performances with audiences. However, compared to scripted sketch comedians who use YouTube as a proving ground [26], improvisers have limited access to the technologies that would allow them to accomplish this co-creation online. For example, simply pointing a live camera at improvisers does not allow for audience interaction; introducing non-copresent audience interaction also introduces the design challenges noted above.

In this project, we seek to understand how new tools for livestreaming improvisational theater can help us understand the design challenges of audience participation in livestreams more broadly. In particular, we focus on extending existing work on audience participation games (APGs) [16, 47] while also supporting the native practices of improvisers. In doing so, we explore both what Twitch could afford improvisers that would not be possible in a physical theater space, and how improv structures could scaffold new interaction paradigms between performer-streamers and their noncopresent livestream audiences.

As a first step to answering this question, we analyzed the features of both domains (livestreamed performances and improv) in order to identify productive overlaps for design. As part of this work, we analyzed existing literature, observed improv and livestream performances, and interviewed domain experts in both domains. We identified both core overlaps and differences. For example, the 
director of an improv performance keeps the show moving, while the same resposibilities are divided across multiple different participants in livestreams.

Based on this analysis, we created a technical system that embodied our insights about livestreamed improvisational game experiences. This system, StAGE-Streamer Audience Game Environmentintegrates green-screen technology and interactive video overlays to scaffold audience participatory improv performances. The system supports role differentiation between streamer/performers, hosts, and viewers/audience in ways that reinforce the norms of both Twitch and improv culture. The system also affords creative communication between a non-copresent audience and live performers.

Next, we created four games for our system, and playtested them in three livestreamed improv performances. We collected data from both improvisers and audiences; the role of host was filled by a research confederate. We found that the system provides novel interactions for livestream audiences, complex audience participation for improvisers, and effective communication between them. Based on our playtests, we also identified key design challenges for livestreamed improv: supporting improvisation, audience presence and social feedback, and hosting. We illustrate their implications for both the intersection of Twitch and improv and existing livestream audiences.

In short, our work contributes: 1) a domain analysis of livestreaming and improvisational theater, 2) the design of the StAGE system, 3) four novel games designed for StAGE, 4) insights from using our system in an authentic performance context, and 5) additional design challenges that are particularly important for livestreamed improv, compared to other types of livestreamed performances.

\section{RELATED WORK}

\subsection{Improvisational theater}

Improvisational theater is an unscripted, group-based theatrical performance that develops characters and stories in a series of often unrelated scenes [24,33]. In a typical setting, live improvisers on a stage create a unique, never repeated performance by playing off both one another's implicit cues and the audience's reactions. Audiences are also given structured opportunities to provide input, such as suggesting scene locations or character traits, to which performers respond. This input is often only used to begin a scene. While improv is often performed in theaters, it can also be understood more broadly as a set of skills enabling collaborative creation between two or more parties [8].

Recent commercial attempts to offer non-copresent improv in virtual reality reduce or remove the audience's ability to collaborate with each other or performers. Some examples include audiences and performers as virtual avatars [1], while others offer real-world live video feeds without voice chat [6]. These shows disrupt embodied qualities, such as crowd communication, important for developing a sense of social co-presence between the audience and performers. This co-presence paves the way for a special, shared kind of knowledge that we refer to as an "inside joke". Because an improv show is unique and never premeditated, each show is an ephemeral experience shared only by those present. This creates a sense of shared possession of the experience between the viewers and performers. Performers exploit this quality for the benefit of their stories with specific callbacks that play off previous, never-tobe-seen-again scenes and events [13, 24]. When performers build a scene by calling back to a previous part of the show, the viewers feel like they are in on the joke; both are constructing a shared mental model of the performance [15].

Prior work has enabled improv with technology to create virtual performers. For example, the Improvisational Theater Space used non-human projections [50]. These projections were autonomous software agents designed to act alongside performers while avoiding rigid scripting, a quality improv also avoids. An audience surrogate robot named Pokey has also been used. Improvisers reacted to audience-submitted gestures and phrases performed by Pokey, though audience input was limited to before shows [34]. Elsewhere, technology has also extended performance capabilities. Improv Remix is a mixed-reality system that allowed improvisers to integrate video editing into their stage performances [13]. These existing systems do not allow for live audience participation, but all show the desire of a broad range of stakeholders to perform with new tools.

\subsection{Livestreaming}

Streamers and viewers collectively improvise to create both narratives and stream culture. For example, they develop memes over multiple streams, resulting in each stream's community having its own language of inside jokes and custom emoticons that enhance the show and sense of co-ownership [37, 41]. Audience members are additionally mutually aware of each other, affording them clearer opportunities to interact with each other as opposed to just the streamer [47]. Streamers and audience members sharing their collective culture is an established behavior pattern [14].

Not many viewers feel intrinsically motivated to watch streams because of the ability to simply interact with the streamer or the other viewers, but instead for dynamic reasons like socialization or this development of culture [18]. Their interest must derive from the interactive potential of being involved [17]. Streamers and audiences collaboratively creating culture, gameplay, and social relationships provides this $[16,28]$.

Experienced streamers often express their desire for ways to foster social activities such as conversation or collaboration [16, 19], both representative of involved co-creation. Stream audiences express interest in experiences of showmanship or skill [40]. Streaming has potential to tap these desires and become tandem play with smaller audiences, where both the streamer and audience pulls equal creative weight [44]. Audience participation games, or APGs, are one attempt at tackling this. Recent work has identified design challenges in streamed APGs [16], such as audience agency (their ability to affect the game), and the cognitive burden of splitting attention between watching the streamer and participating themselves. Solutions like the ubiquitous voting system in Twitch Plays Pokémon can alienate viewers through aggregation [29]. More recent methods like asymmetrical audience roles, titles, and abilities are difficult to communicate to users [16]. New interfaces and design approaches are needed to take advantage of this space. 


\subsection{Augmented streaming interfaces}

The current Twitch interface is composed of streamed audio and video, a live chat, and optionally, an extension. Extensions are stream-specific video overlays that allow for audience interactions [55]. For example, audience members can click on an extension to easily view the standings in an ongoing tournament. It is possible to display different views to different audience members, and to have each audience member asymmetrically interact with the extension.

Twitch's live text chat is by itself not an expressive enough option for audience creative participation [29]-this is especially true as chat scales. Small chats have longer, more developed messages; larger chats have reactionary, simple messages, often only emoticons [38]. Extensions and exterior web pages have been used to offer additional creative information or interactions $[16,30]$, though they take up screen space. The video feed is the most important part of a stream, and obscuring or condensing it must be considered if it is worth it [48].

\section{DEFINING KEY DOMAIN FEATURES}

Identifying critical elements of both fields beyond the existing literature was critical to our design process, as these findings directly informed our system design. To understand improv, we attended six shows in two different cities. The skill level of the performers ranged from students to hobbyists to professionals. After each performance, we debriefed as a team, focusing on similarities and differences between performances, crowd engagement, and mistakes. Four team members attended weekly improv classes throughout the duration of the project; one team member is an established improviser and provided expert insights. To understand Twitch, we viewed popular streams daily for two weeks and weekly thereafter, met with expert researchers to discuss their work, and studied interviews with experienced game designers and producers who have worked on APGs $[4,35,42]$.

\subsection{Social structures}

The overall social structures of livestreams and improvisational theater are similar. First, both have improvisers who create live performances. In improv, these are actors who play scenes in response to audience suggestions $[50,51]$. In streams, these are streamers who give live commentary on their play [10] and respond to viewer actions such as donations or messages [5, 61].

Second, both have audiences, who watch the performance in real-time. In improv, audience members both watch and contribute suggestions that help the performers improvise [50]. Typically, improv audiences attend shows in person. Stream viewers, like improv audiences, watch streamers' live performances. In APGs, they contribute material as improv audiences contribute suggestions [16, 24] However, unlike improv audiences, they are typically not copresent with the performers. Instead, they communicate with streamers through a text chat or services such as Discord [16, 22]. While viewers have many different motivations for watching a performance $[7,49]$, improv audiences are typically engaged or participatory $[24,50]$ and APG design has in part focused on encouraging viewers' participation [16].
Finally, both livestreams and improv performances have roles dedicated to keeping the show moving. In improv, this is a director [51] who ends scenes that are going poorly, amplifies the best parts of the performance, and frees the improvisers from other show-related responsibilities. For an example of these responsibilities, Drew Carey hosts the televised improv show Whose Line Is It Anyway? [39]. He accommodates performer introductions and exits, commercial breaks, and rule explanations; he also contextualizes the performance and explains any required knowledge for a broader audience less experienced with improv. For improv that runs without a formal director, a performer will temporarily take on all of these duties when necessary [11, 23, 24, 53].

In livestreams, this type of logistical role is divided across multiple different participants. Moderators help streamers manage their community. They curate audience content, socially engage with viewers, and enforce community rules [45]. Bots are often used to provide information about a stream, such as what music is playing in the background or what game the streamer plans to play next [46]. Event-based livestreams, such as competitive fighting games or talent shows, also have commentators, comparable to sports broadcasters. These individuals explain what is happening in the stream, call out particularly interesting moments, and help interpret the action for viewers of varying experience levels [3].

Across these varying contexts, we identify this social role as a host. In improv the host is typically one individual; in livestreams, the role can be collaboratively filled by one or more humans and/or one or more bots. However, the goals of the host role are the same: to support the performers in effective improvisation, and to manage audience understanding and/or interaction.

\subsection{Audience understanding}

Livestreams also have structural elements that communicate synposes and ludic contexts implicitly, as opposed to the explicit help of hosts. Many streams occur on regular schedules with predictable formats; for an example of the latter, successive rounds in a competitive game. This foreseeable nature aids viewer understanding on a platform where dropping in and out of shows is common [16]. In improv, though, it is typically not socially acceptable to arrive late in the middle of a show [53]. Improv's linear norms let performers make callbacks in front of an audience that has all the necessary information to understand [24]. This difference has implications for designing improv experiences in a drop-in, dropout context-implicit methods of helping the audience understand improv contexts will be necessary.

\subsection{Co-creation and participation}

The interactions between regular stream viewers, and their corresponding generation of content such as emotes and memes, is a parallel to the scene development and recurring callbacks in an improv performance. In both contexts, there is a special, shared knowledge between the audience and performers that results in these creative interactions [15, 19]; this joint mental model [32] is collaboratively and ephemerally composed of inside jokes. In improv, inside jokes are called back to, but only across scenes in the same performance. Each new performance (with the same or different performers) generates its own, unique inside jokes. In 
livestreams, inside jokes can develop beyond a single streaming session, since the channel is perceived as a stable "third place" for community to develop $[19,20]$. Contrary to improv, normally passives users may perceive less creative involvement [19]. In both mediums, inside jokes are a critical way for performers to show mastery and for audience members to feel a sense of belonging.

Their respective inside jokes are developed in substantially different creative atmospheres. Theaters are politely laconic, while livestreams are chaotic and talkative. Audience laughter is strong social validation in improv theaters [23], but limited to text and emoticons in live text chats. Alcohol is served in many improv theaters, while livestream audiences must serve themselves, if at all. Audience members in a theater are physically copresent, while in a live chat only those who participate are perceived. Improvisers are physically copresent both with the audience and with each other, while streamers often work solo. Improvisers and streamers expect to communicate with an audience with these respective qualities $[24,60]$. These differences must be taken into account when designing for this space.

In both mediums, participation is often rewarded with social validation. Improvisers thank audience suggestions. Streamers acknowledge members of chat by directly reading their comments, showing gratitude for donations [5, 61], and appointing moderators [45]. A performer or streamer validating one audience member lets others perceive a potential for social validation themselves. Social validation, especially when given in front of a crowd or by admired figures, is important to creating an engaged, participatory audience $[21,24]$.

\subsection{Latency and flow}

One pragmatic difference between livestreaming and improv is platform latency. In improv, audiences and performers are copresent, so audience contributions and actor responses are near-instantaneous. In livestreaming, the latency (an average twelve seconds on Twitch [62]) between an audience member engaging with the performance and the corresponding response can affect comedic timing, tension, and a streamer's adaptation to their audience. Latency is a disjoint in how performative information can flow between the audience and performers. One approach to addressing this issue is asynchronous interactions, such as a round delay of inputs [16]. However, designing with hosts in mind offers a new set of solutions, as we describe below.

\section{SYSTEM DESIGN}

We used these insights and observations to develop StAGE, a system to support livestreamed improv games. This system lets experienced improvisers both augment their performances with virtual, interactive elements and communicate with non-copresent audiences, while also affording new interactions for audience members compared to both Twitch and traditional improv. Our system is organized based on the different social roles we identified (see Figure 1) in both livestreaming and improv. We now describe each element in turn.

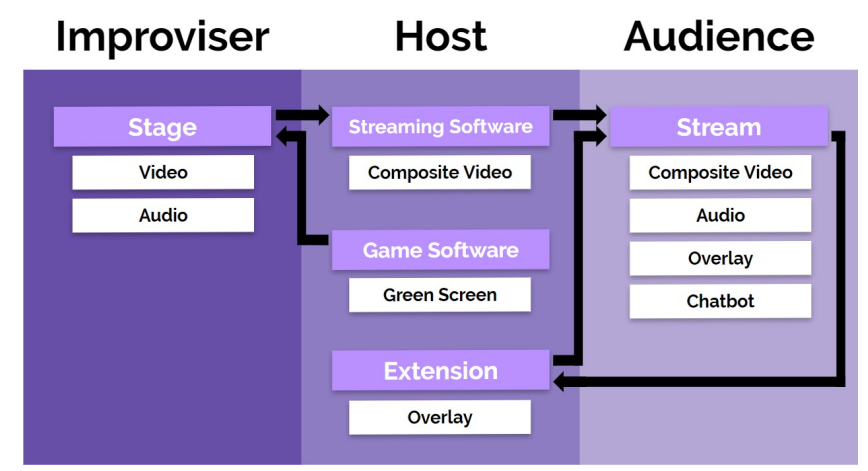

Figure 1: StAGE - $\underline{\text { Streamer }} \underline{\text { Audience }}$ Game Environment.

\subsection{Improviser}

For improvisers, our design goal was to capture their performance for streaming, while giving them opportunities to react to virtual elements and a non-copresent audience's input. We designed for experienced improvisers to focus on the system's potential to augment performance, not create a performance itself. We created a physical space for improvisers to perform co-presently, while audiences watched remotely, such that the focus of the streaming system would be on audience-to-performer interaction rather than performer-to-performer. We captured audio and video feeds of the stage, which were composited by streaming software and livestreamed on Twitch.

Improvisers receive filtered information. When audience members provide input, either an algorithm or the human host selects or summarizes the material before they are communicated to the performers, depending on the game. The curated material is then communicated to the actors and general audience simultaneously. This simultaneity is designed to foster a sense of co-ownership and to not lose human timing. The improvisers can receive this information verbally from an off-stage host, or through visuals on a large monitor or audio from speakers copresent with them; these latter two outputs also support the interactive virtual elements of our improvisation. Finally, the entire space was set up with a green screen background, allowing improvisers to perform in front of composited images and provide implicit visuals to aid viewer understanding.

\subsection{Host}

Hosts enable improvisers while coordinating audience contributions. In our system, hosts control the streaming software, the game software (e.g. the green screen), and the extension running on the stream itself. All three interactions are controlled from a computer visible from the improvisers' space. As described in the previous section, being copresent allows instant communication between the host and the performers, without latency. Because of this, all audience contributions are communicated by the host, as we describe below. The audience is made aware of the host by the performers throughout the performance to ensure their understanding of offstage voices and transitions. They are also instructed to ask the host any questions about the technology, the games, or anything else. 
The host could respond via the steam's live text chat, and further acted as a moderator.

The game software is similar to a show controller, an automated system used in theaters to control elements such as lighting, set movement, and curtains [9]. Our controller modifies the green screen behind the actors, which displays contextually relevant information. This can be used to provide viewers dropping in on a performance already underway a passive, immediate context to narrow their sense-making. During our prototype games (see below), the green screen shows the background of where a scene is set, such as an airport or a courthouse. Between games, the green screen displays procedural information to the audience, such as what game is coming next, where to check for rules and research forms, and other logistics. This is also done to aid the understanding of viewers dropping in.

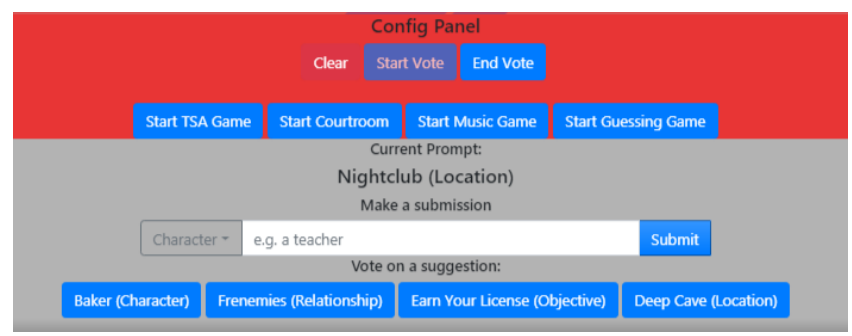

Figure 2: A cropped view of the extension overlay that appears at the bottom of the video feed. Audiences would interact with the section in grey, while only hosts could view and interact with the section in red. See Figure 3 for a full audience view.

The extension is an interactive overlay that appears on top of the audience's video stream [55]. It enables the audience to participate in the games without derailing the chat and disrupting the creative atmosphere [30,47]. Most of our games' software was housed within the extension, handling both audience input and video overlay output. While viewers used the extension to participate (see below), the host used it to communicate with viewers and curate content. For example, the host collected audience inputs from the extension, used the data to select material for the performers, and updated the extension with new data between states, such as between a voting period and a submission period. This was vital for creating a sense of co-creation and bypassing latency.

\subsection{Audience}

The audience watching the stream on Twitch received the composite video and audio from our streaming software, along with the extension. The extension overlay was designed to allow user interaction without distracting them from the performance, and was therefore minimalist in its visual design. The audience could interact with the overlay by clicking buttons, following links, or entering suggestions into text boxes. Relegating nearly all interactions to the extension allowed for responsive, viewer-specific feedback and an uncluttered chat for conversation [30], though certain games benefited from moving text submissions to chat. Audience submissions were socially validated through text feedback that also communicated how their information could be communicated to the performers to ensure understanding.

\section{GAMES}

\subsection{Game Design Methods and Development}

Based on our research goals, we developed a set of game design criteria. First, each game had to use StAGE to expand an existing improv game with novel, participatory interactions. This would allow performers and hosts to more easily use their prior experience to run successful shows. Second, audience input methods must be diverse enough that we could learn something new from each game. This was done to explore a breadth of involved interactions, as described in our Literature Review. Third, performance information must pass between the audience, the host, and the performers. While this criterion manifested differently in each game, it allowed us to evaluate how StAGE would allow each game to approach our described challenges such as latency, flow, and non-copresence to create a successful performance.

We generated a list of improv games that were potential candidates to adapt to StAGE. We collected games from established shortform improv genres [13, 24], such as warm up games [51] or Theatresports [24], because of their lower complexity, shorter runtime that allows for more opportunities for input, and clearer rulesets compared to long-form improvisation [23, 24]. We also prioritized based on our own experience performing them. Next, we generated a list of possible audience inputs mechanics, drawing from existing APGs such as Twitch Plays Pokémon's voting [27] and established improv suggestion methods. We ideated potential prototypes by juxtaposing items from these two lists, pairing improv games with new participation methods, and then ordering the pairs by their adherence to our design criteria. We developed the top four games from this list.

The audience input element of the games was created with Twitch's developer tools. The show controller, designed in Unity, was used by the host during certain games to affect the stage visuals and display audience input. An IRC-based protocol allowed user text chat to interact with the extension in addition to its on-screen inputs. All games were collectively developed by our team over fourteen-weeks.

\subsection{Freeze Tag}

Our first prototype was a proof-of-concept to test a simple introduction of audience input. Success meant having live audience input be communicated to a performer during a show. In improv, Freeze Tag is a common warm-up game $[25,51]$ where two improvisers perform brief scenes, focusing on body movement and quick characterizations. At any time, an off-stage improviser may call "freeze!" and pause the scene. This is done when the scene has either reached a comedic peak or is going poorly. This off-stage improviser then enters the stage and tags out one of the frozen performers, taking their place and body position exactly; the tagged performer exits. The new improviser then starts a new scene that justifies their incoming pose. The other on-stage improviser follows and builds the new scene with them.

Our version introduced text-based secrets submitted by the audience that the incoming improvisers would use to start their scenes 
in addition to a pose. Audience members can submit any number of secrets. They select one of four types of secret to submit, and enter their suggestion into a text field on their video overlay. Those four types-Character, Relationship, Objective, Locationguide audiences to provide material that is easier to work with. It is difficult for improvisers to work with a vague and broad secret like "milk", but easy to establish important scene elements with a more actionable one like "farmer" [24].

StAGE was used to implement the input method and the communication of that input to the performers. Whenever "freeze!" is called, four audience suggestions are randomly pulled and displayed as a poll for the entire audience to vote on. Audience members make their selections anonymously through StAGE, and once voting is ended, the host prepares the entering improviser with their secret. The host is responsible for syncing voting periods with the performance timing to avoid latency; they are also constantly curating what suggestions are selected for the poll through actions like censoring obscene suggestions or removing repeated submissions. To aid audience understanding, participation is a choice, allowing audience members multiple interest curves [43] to pick up or fall back on.

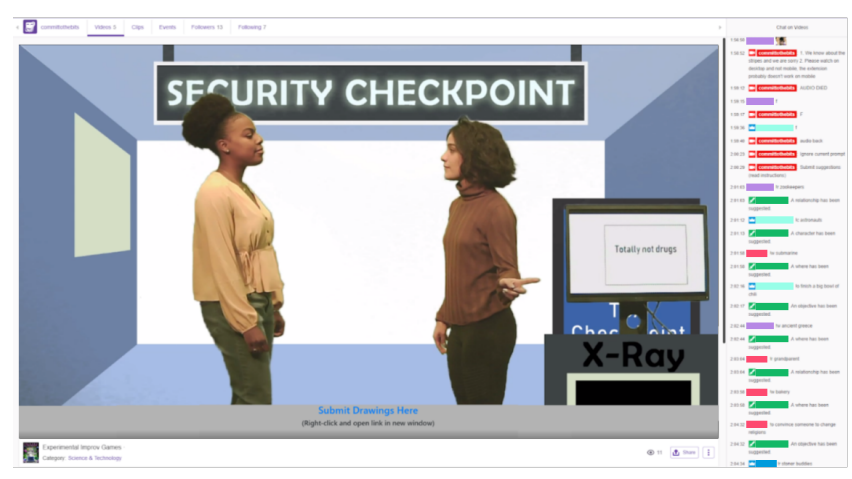

Figure 3: Audience view for Whose Bag Is It Anyway? The overlay is at the bottom of the video feed.

\subsection{Whose Bag Is It Anyway?}

One performer takes on the role of an airport security agent, and the other, a traveler. Audience members suggest suspicious items to be "found" in the traveler's luggage. The traveler must attempt to explain these items to the security agent in a way that creates an emergent, humorous narrative. After about three items, the scene ends, and a new one begins. The previous traveler becomes the new security agent and a new improviser enters as a new traveler.

In live improv shows, audience suggestions are limited to verbal descriptions. After designing text-based input in Freeze Tag, we wanted to explore image-based input instead. In this game, successful play meant improvisers creating scenes from images as opposed to words. Audience members submit images by following a link displayed on StAGE to a public Google Drive folder. StAGE allows for this input novel to normal theaters. Images are encouraged by the host to range from crude, digital scribbles to previously existing images found online. The host curates submissions into a queue, censoring if necessary. When the performers are ready for a new image, the host selects one from the queue. For example, the host might choose a picture of an illegal item after the traveler says they would never bring illegal items to an airport. The image is simultaneously shown to both improvisers and the audience on a physical monitor in the space, mocked up as an X-ray machine. StAGE further reinforces the scene's passive context with its green screen background, which displays an airport.

\subsection{Discourt}

One performer takes on the role of a defendant on trial for an unspecified crime, the other, a prosecutor. Audience members act as witnesses brought to the stand by the prosecutor. The witness accuses the defendant of a crime; this accusation is unrelated to prior accusations and taken as truth by all performers. The defendant must justify and connect each accusation, collaboratively telling a story between the actors and audience. After a satisfying story is told, the host shouts a verdict at the defendant from off-stage and transitions to a new scene with new performers.

While improvisers become unique characters in each scene, audiences are less directly involved. They do not transcend their role as an audience member by becoming a character. StAGE allows this game to bring viewers directly on stream, with performative roles equal to those of the actors, through calling in with Discord. The host pulls witnesses from a queue on StAGE open to all audience members. These viewers become guest stars represented by an animated avatar, a penguin witness, that is puppeted with StAGE by the host and displayed on a green screen behind the actors. This puppet appears between the actors, and has animations for entering and leaving the courtroom as well as for speaking. The host matches the puppet to the users' actions and voice. Successful gameplay results in audience members seamlessly "coming on stage" with the performers and improvising together.

\subsection{Music to My Peers}

Traditional improv uses music sparingly and passively; for example, to provide a varying tempo or as a shortcut to an emotion [24]. However, Twitch has a strong music culture, seen in elements like the official Twitch Sing's karaoke system [57] and established tools for background song queues filled by viewer donations [31, 36]. We designed Music to My Peers to bridge the gap with StAGE using viewer-selected music to modify scenes.

This game is structured the same as Freeze Tag, but a music cue replaces a shouted " "freeze". Two performers on stage verbally ask the audience for a secret, such as requesting a Character, and wait for a response from chat that is then selected by the host. This was done to examine how the host directly reading chat could affect how performers received information. After receiving it, they start their scene. A different type of guest star, a DJ, is selected by the host from a sign-up queue on StAGE. The DJ is then provided three random selections from a pool of generic music genres, such as Romantic, Tragic, or Heavy Metal, and selects one. This is a specific ask that narrows their possibility space better than open-ended prompting [23]. This process repeats until they have created a "playlist" of three songs. DJs are also encouraged to poll the audience to see what genres the audience wants, ideally involving the crowd and putting the DJ into a position similar to an improviser. 
When the scene reaches a point where "freeze" would be called, the host instead changes the music according to the DJ's playlist, and allows the scene to continue. The performers respond by changing the mood and tone of the scene to match the new music. For example, if the scene were at an office between two janitors when romantic music began to play, the performers could respond by suddenly becoming janitors in love with each other. The host can choose to slowly fade between songs or make sharp cuts. Once all three music choices are finished, the improvisers wrap up the scene. New performers take the stage to start the next scene, and a new audience DJ is selected.

\begin{tabular}{|l|l|l|}
\hline \multicolumn{1}{|c|}{ Game } & \multicolumn{1}{|c|}{ Question } & \multicolumn{1}{|c|}{ Mechanic } \\
\hline Freeze Tag & $\begin{array}{l}\text { Can hosts use StAGE to get information } \\
\text { from the audience to the performer? }\end{array}$ & Live voting \\
\hline Whose Bag Is It Anyway? & $\begin{array}{l}\text { Can audience members suggestions } \\
\text { work as images instead of words? }\end{array}$ & Image submission \\
\hline Discourt & $\begin{array}{l}\text { How can StAGE allow for audience } \\
\text { members to bypass latency? }\end{array}$ & Voice chat \\
\hline Music to my Peers & $\begin{array}{l}\text { Can StAGE let audience members guide } \\
\text { audience participation? }\end{array}$ & Audience-guided voting \\
\hline
\end{tabular}

Figure 4: Each of our games tested a different element of StAGE through supporting a different mechanic.

\section{PLAYTESTING METHODS}

\subsection{Performances}

To playtest our games in an authentic context, we hosted three hourlong external performances with experienced improvisers $(N=7)$. The performers were compensated and drawn from drama schools. We chose performers with experience with copresent improv because our focus is to test StAGE's potential for non-copresent audience participation in improv, and not for educating novices. They performed our games on stream for a non-copresent live audience $(N=36)$ drawn from existing campus research pools. Members of the research team served as hosts, copresent with the performers; we describe this role's selection criteria below. The streams were public on Twitch, but a consent form was necessary for viewers to play the games, interact with performers, and have their data collected. Performances averaged 16 total viewers and 7 "active" viewers (defined as sending more than 5 chat messages total), with a peak of 21 viewers. Both performers and audience members had a range of experience with livestreams, ranging from none to extensive practice. Freeze Tag opened all three performances as a short warm up for the performers and to acclimate viewers to the interface. We then tested two additional games in each session. Since we chose shortform improv games as our basis, as described in Game Design Methods, each playthrough of the post-Freeze Tag games lasted around 5 minutes. Each game was played 4-6 times, per the audience's interest, with a 5-10 minute performer break between games. This rhythm is common in shortform improv [24]. We recorded video, audio, and text chat logs of each performance.

We evaluated the participant experience of StAGE as follows. After each stream, audience members were surveyed on the games and show itself. All 12 active users participated in the survey, with a total participation of $33 \%$. To examine their sense of co-ownership, we asked users if they felt they had made an impact on the performance and for any desired methods to influence the show. To examine their understanding, viewers were asked to explain times they were confused and how they tried to resolve their confusion, such as by asking the host. Finally, open-ended questions were asked about the games' interactions and show structure. Performers were also interviewed individually after the first performance, and as groups after the subsequent performances. They were asked questions regarding their perceived relationship with the audience, such as how they understood the audience through the host or what Twitch took away from the performance's flow. These questions illuminated their opinions on co-creation. Open-ended questions were also asked about difficulties of performing with the system and differences from normal improvisation.

\subsection{Data Analysis}

To understand our playtest data, we drew from the audience text chat logs, audience surveys, and performer interviews. For the chat logs, we analyzed chat volume, comments that sparked discussion in chat, and questions asked by viewers. In the surveys and interviews, we coded data into one of two categories: feedback on game design and feedback on the performance experience. In the first category, we primarily looked at interaction descriptions, including both interactions performers and viewers had and ones they desired. In the second category, we coded answers that illuminated the relationship between the performance we provided and traditional improvisational theater. Cumulatively, we identified what role in StAGE each feedback item originated from, related it to feedback received from other roles in similar interactions, and derived our insights from the resulting horizontal view of the system.

\section{INSIGHTS FROM PLAYTESTING}

\subsection{StAGE and Improvisation}

All games except Discourt (discussed below) were successfully performed and completed by our improvisers. Per the repeatable nature of the games, we define success as being performed in full, multiple different times, with multiple different improvisers and audience prompts. The performers enjoyed the structures for receiving submissions, and praised the novel variety of ways the ideas were presented e.g. images, songs. Performers misinterpreted about one suggestion per game, but this once led to the peak of a Whose Bag? performance: an actor could not identify a picture of a brown egg, calling it an "orange ball," much to the confusion and then humor of their stage partner and audience. Every show also had at least one miscommunication with the host, but these situations were clarified by either the audience or the performers.

Our improvisers found acting off a green screen difficult, with one (P3) describing that it could cause "unsureness" in their performance due to a lack of "clarity" when relied upon in Discourt. This may be an established skill for film actors, but it is a new one for the average improviser, who is trained to build off physical cues [51]. This is just one example of how improvisers will have to learn new skills to operate in a streaming environment. As another, our improvisers asked in every performance to view the chat directly. We view this as them wanting to situate their performance in their previous, direct experience of understanding their audience. While 
they were happy to have the host single out interesting messages and handle unwanted suggestions, they resisted having the host socially interact with the chat for them.

Our participants reported enjoying an expanded range of audience interactions, even when those interactions were only novel for improv. For example, voting is an established interaction in livestreaming APGs [27, 29], but not for improvisers. All actors we interviewed said voting was an exciting, novel interaction with their audience. Further, they enjoyed the variety of suggestions to perform with, particularly the ability to receive images, when compared to the traditional suggestions shouted from a crowd. Unilaterally evaluating the novelty of our experiences is therefore difficult; for example, Whose Bag?'s image submissions are novel to improv, but derivative of commonly livestreamed games such as skribbl.io [2]. The involved, co-creative aspects of improvisation remain the most novel to livestreaming, and StAGE succeeded in bringing that to our audience as described below.

Our surveys uncovered that audience members wanted more control over what was going on in every game. While the majority $(83 \%)$ of our surveyed audience felt they had a realistic potential to impact or control the performance, all still wanted more breadth to their inputs. Specifically, the audience expressed desire in chat and in surveys for more of a "choose your own adventure." They wanted to mix and match actors with specific characters and scenes in Freeze Tag, submit their own music in Peers, or select what game was next and with what context, such as changing the StAGE background in Whose Bag? It is challenging to balance the necessary responsiveness of more direct control like this with the inherent latency and necessary comedic timing. All input tested involved the host to handle these two challenges, but future performances will need to account for audiences' desire for less restrained creativity by extending StAGE.

\subsection{Audience Presence and Social Feedback}

It is difficult to adapt social feedback virtually; social presence, or crowd feel, in a non-copresent stream and its chat is fundamentally different than that in a theater and its copresent audience. On the one hand, audience members could rarely hear anyone laugh. In two performances, off-stage performers or hosts would laugh audibly and be relayed by the microphone, providing social feedback to both audience and performers. This prompted viewers to say that they wanted to hear more laughter. They reported wanting to hear laughter as a reward for their own participatory actions and for the actors to hear that the audience was entertained. On the other hand, interviewed performers reported that the lack of a copresent crowd was comforting in a way, that it lowered their anxiety of performing. They still believed, though, that there was overall less social validation and feedback from this non-copresent audience than that from a copresent audience. This offers additional insight on their desire to directly see the chat. Performers adapt their performance to their audience's reactions, and here it was difficult to do so. Both the actors and audience members benefit from feeling like they are in the same, embodied space-StAGE provides this in part through its hosts, as we describe below.

Audiences responded positively to seeing their names across our games, such as on winning submissions in Freeze Tag or the
DJ names in Peers. We noticed an increase in chat activity when improvisers or the host were directly thanking audience members for their submissions, though this requires further testing. While this social validation was noted by the audience as valuable feedback for their own performance elements, such as suggestions and chat messages, the most negative reaction to our system was its lack of process feedback on inputs. 3 audience members reported being unsure, for example, of how much their vote counted. StAGE primarily operates on text feedback, such as confirming a submission with positive text appearing over the completed input box, but there are multiple elements of feedback we have not explored, such as broader audio and visual feedback or personal submission tracking. Twice, audience members asked the host to assure them their inputs had the potential to affect the performance.

\subsection{Hosting}

The role of the host is vital to running livestreamed improv shows for two reasons: communicating performative information and ensuring audience understanding. These alleviate some of the issues described above with non-copresent audiences. During our playtests, hosts communicated performative information to both sides by monitoring, parsing, and curating both the chat and submissions. In terms of chat messages, this ranged from audibly reporting the audience's feelings on the current performance to sharing funny audience commentary on the actors or scenes. Communicating messages like these gave performers relevant feedback to adapt their performance; it also socially validated both sides' performances where crowd feel normally would in a theater. In terms of audience submissions, the host can both intelligently censor inappropriate submissions and subjectively select good submissions to ensure performance relevance. In this way, StAGE lets the host act as an improviser as well, interjecting their curated information as conversational elements that change the scene. The performers reported that the host was a valuable, communicating member of the improv team.

The host can also reduce the cognitive load on the audience. At the start of all games except Discourt, the host would show the audience how the system worked with example submissions and results. From these behaviors, the host acted as a model improviser that communicates a clear interaction paradigm for audience members wanting to participate. They understand how their information can be filtered and how to present it. The host offered further help to anyone in chat who has problems or questions, which is especially important in a system that has a) flexible rules not fully explainable by a static document and b) potentially unseen technological asks, such as signing participants up for a Discord account. Feedback from the audience on the host reported they were a positive, natural, and most importantly non-confusing show element.

The host both alleviates latency that normally impacts a necessary sense of co-creation and ensures audience understanding in a drop-in, drop-out context. Their tasks in doing so, from the intelligent curation of content to explaining flexible rules, are overall not viable with an automated system, but are with a human, a host, behind it. 


\subsection{Additional Challenges}

7.4.1 Audience Understanding. StAGE had several mechanisms in place in order to facilitate audience understanding, especially for those who would drop in during the middle of a scene. We used the green screen behind our performers to superimpose them on various backgrounds, giving new viewers an immediate understanding of what the game was about. For example, in Whose Bag? we placed the performers in an airport security checkpoint, which signaled the roles of the performers to viewers. Discourt similarly had a courtroom. Further, the green screen allowed viewers to understand when the Discourt guest was speaking through the use of the puppet instead of being a disembodied voice. Surveyed audience members reported that these contextualizing features were fun, non-distracting additions to the game, with one commenting specifically on their quality use as context clues. The majority $(67 \%)$ did not comment on their ability to aid in their understanding, we believe due to the majority watching the performances in their entirety from start to finish.

StAGE also had a toggleable Instructions tab in the extension that showed the rules for the current game that was being performed. These rules were always repeated by the host or a performer at the beginning of each game as well. Most viewers listened exclusively to the verbal instructions from the performers. The most used sensemaking aid was the host's presence in chat. By having the host always on standby, any questions about using StAGE's extension, what was going on, or bug reports were dealt with in real time. Participants said this was a beneficial, human element of the show that improved their enjoyment and comfort.

7.4.2 Barriers to Entry. For every experience we created, there were multiple barriers to entry within StAGE or our games that impeded the audience from experiencing our content. We divide these into two categories: technical and social.

We begin with the social. While a Twitch username offers some level of anonymity, usernames can still identify a viewer in a way they may not be completely comfortable with. Some users may not participate if a submission is tied to their username, while other users may prefer their name on what they submit for further social validation. We tested one game, Whose Bag?, with unsigned submissions, the rest, signed, and did not come to any conclusions. Based on our feedback, both types of user are present, and need to be accounted for in future performances. Furthermore, the prototypes we developed gave viewers varying amounts of responsibility to entertain the rest of the audience. For example, in Discourt, participants had a very high responsibility to entertain because they were expected to speak directly to the performers to build on the scene; however, their username was never exposed during the game.

Technical barriers depend on the various implementations of our games with StAGE. They are primarily events where the user must provide an input to the game; for example, by clicking on an onscreen button to vote in Freeze Tag. There is less effort in pressing a StAGE button (Peers) than there is in submitting a specific image (Whose Bag?), and even less than there is in making a voice call over Discord (Discourt). When testing Discourt, only one audience member entered the guest star queue, but stopped responding after becoming lost in the Discord call-in system. No audience member participated in the game. The technical process of creating a Discord account, adding the research account as a friend, and answering a call request was lengthy, and the user reported they became lost and confused. We found that the lower-effort StAGE interactions, such as voting or text submission, generated more participation than the higher-effort interactions, such as calling in or becoming a guest star.

\section{DISCUSSION}

Glickman et. al. model livestreams as a distributed digital hearth where audiences form for togetherness, social connection, and appreciating the performance of both streamer and other audience members $[16,59]$. Gameplay is the central, expressed reason to watch live, and meaningful participatory gameplay has potential to bring audiences closer together. [16, 47]. Elsewhere, audiences attend improvisational theater to witness a creative process, vicariously perform, and ultimately also appreciate performance $[23,50,51]$. There, improvisational acting is the reason to attend, not to be social. It follows then that to combine these mediums, we must strive for participatory improv that blurs the line between audiences and performers. As described, these two may naturally solve some of their design challenges-like adapting social roles of improv to ease audiences' latency and understanding, or technical livestream interfaces that create novel, participatory improvisational interactions-but questions still remain.

\subsection{Embodiment}

As related work established, improv theater is most commonly a physical commitment requiring present audiences in a real theater. To reduce our scope of study, we chose to have our actors copresent. A performance being livestreamed, though, potentially makes copresence unnecessary. If audiences can be non-copresent and still be able to create a shared experience $[12,19]$, what theatrical elements must be translated to create a shared performance? We will discuss body language and simultaneous communication.

Performer-to-performer communication is often necessarily subtle through interactions like a raised brow or small shoulder tap so as to not disrupt the show $[8,23,60]$. Body language like this is perhaps the least transferable element of an embodied performance; it is less noticeable and effective when between screens instead of in person. If the actors were non-copresent, a central host observing the scene could distribute information instead, but collating all the performers' bodies would be difficult.

Copresence between actors and host, also ensures fast, simultaneous communication and understanding. This fosters good comedic timing. Improv does not work without this timing, and this communication is impeded by platform latency [62]. Because the host in StAGE is copresent with the performers, communication is instantaneous, which allows them to interject audience input at proper times. If the current system were to be modified to have the host composite non-copresent performer streams, this instantaneous communication would be lost between all, along with comedic timing. This would disrupt the flow of the games, interrupt the performers' understanding of the audience, and distance the audience from the action more. Video latency will resolve itself over time as technology improves, but remains a design issue preventing non-copresent, embodied performers. 
A possible source of crowd feel for a non-copresent audience is to mix the virtual audience with a live audience. Similar to how television shows can be filmed in front of a studio audience, an audience copresent with the performers could provide laughter and corresponding feedback to suggestions and choices submitted by a virtual audience watching the same show. Our interviewed audience members wanted this type of feedback. There is also a potential to naturally split users into two types: those who wish to interact could participate with StAGE, and those looking to be passively entertained could attend the show. Existing theatrical experiences have used virtual reality for spatialized audio and visuals [1], but lose out on body language. We believe the presence offered by virtual reality could be a solution to audience crowd feel, but translating StAGE to the new interaction space would be necessary.

\subsection{Extending StAGE for Understanding}

As mentioned, performers wanted to read chat directly. They want to be able to understand their audience to receive social validation and adapt their performance. Reading chat directly is not viable for multiple reasons: delaying performance time, ignoring the presence of other actors, and dividing attention among viewers. There are better ways to accomplish this; improvisers must learn to receive feedback in different ways. Instead of forcing actors to parse text comments, pictures, or emotes while performing, our use of the host turned the process into a human-to-human conversation. As described, filtering performative information through the host alleviated latency, timing, and audience isolation. The delivery of this information must become conversational and improvisational to function naturally in a performance. Additionally, StAGE itself could be extended to assist with the actors' interpretation. A billboarding tool that would allow the host to directly show performers specific images or messages from audience members would be a useful supplementary option; games such as Whose Bag? indirectly support this feature, but only have the host displaying audience submissions in the game's context. This tool, if visible to the audience, would help present the information simultaneously to the audience and performers, in turn promoting a sense of co-creation.

When livestream audiences scale up, chat becomes faster and audience understanding harder to maintain, causing socialization and participation to become more difficult $[19,38]$. The shared, participatory experience risks being lost in larger crowds [16, 30]. In StAGE, this experience is underpinned by the host. Our games were not tested at the scale where Twitch chats tend to lose this feeling, around 150 viewers [19]. A single host could not keep up with chat sizes in the hundreds. While StAGE was designed with one, central host in mind, the system could be extended in order to help the host manage scale. Elements of the presently manual show controller could be integrated to the states of the extension and its games to lessen the operational load on the host. The system could also support recent Twitch features. Twitch Rooms [56] could let audiences split themselves up, differentiating their roles for the host and making queue management easier. Squad Streaming [58] could allow for each performer to have their own host managing their own subset of the audience, and could also potentially allow each performer to get their preferred amount of interaction with the audience as was wanted by our interviewees. Moderators and bots can also aid in communicating with a large crowd [45, 46]. By decentralizing and reducing the load on a single host, the system can be improved to have a more efficient flow of information to keep performers and audience members improvising.

\subsection{Social Participation and Audience Agency}

Long-running "inside jokes" were difficult for us to investigate due to our limited and relatively inconsistent viewership. Still, a success of our games was having viewers consider themselves performers involved in their creation. In livestreams, this is a large difference from a normally passive viewer $[19,47,49]$, and we believe it to be caused by StAGE's imported improv social structures. The communicated and socially validated role of audience participant (guest star, voter, submitter, etc.) bolstered audience confidence in taking responsibility for their own entertainment; an interviewed viewer (A7) participated for the opportunity to "look smart" in front of the audience. This created more collaboration with the actors, and helped establish a sense of co-ownership between them. The interaction paradigm of the host further communicated how and what they should do.

What needs to be done is to naturally pace when and how audiences become performers. Our games are dependent on willing participation, and at times had to take what they were given. Participation, and therefore engagement, does not have an interest curve that balances high-intensity interactions and low-intensity downtime [43] that accommodates the varying audience desires to participate. In our short playtests, this was not a problem, but in longer streams that may repeat games over time or in longer-form improv, audiences will need to have their experiences designed with pacing in mind to not burn out on their performance, just as actors need to take breaks from time to time.

There was a lack of malicious trolling or obscene suggestions, behavior observed as "hindering" in APGs [47]. This was fairly unlike improv shows where audience members often say edgy suggestions in order to draw attention to themselves [51]. We believe this is partially because of their awareness of the host's curation, and partially because performers socially validated cleaner suggestions. The established sense of co-ownership of all audience ideas may have reduced these, though this must be tested at scale in the future.

\subsection{Hosts as Improvisers}

To be a host, one must also be an improviser. The host must be familiar with the game rules, content each performer works well with, and most importantly proper timing. The latter because one of the host's largest roles in the performance is keeping it going, and knowing the points at which to cut, move on, or introduce audience input is important to that. Therefore, a host should have similar practice to that of an improviser. Group teambuilding efforts that improvisers undergo are the most useful when learning the rhythms and patterns of their performances. Based on our internal playtests, it is possible to have performers rotate between serving as the host in terms of controlling the extension, but this could quickly confuse audience members if they were unfamiliar with the vocals of certain hosts. 


\section{CONCLUSION AND FUTURE WORK}

In this paper, we establish key domain elements of livestreamed improvisational performances. These four features-new social structures, audience understanding, co-creation and participation, and latency and flow-are vital to consider when creating improv in a virtual space, and applicable to other types of livestreamed embodied performance. We present StAGE, a system for creating livestreamed improvisational performances, and how it accommodates the flow of information between performers and a non-copresent audience. We designed four games with this system that explore novel affordances of livestreamed improvisational performance. We report playtest insights from performances of these games from both an improviser's and audience's perspective, establishing that our system can indeed operate in this domain.

StAGE is just one implementation for livestreaming improv; our work does not touch on the informal improvisation found in formats like live adult entertainment or non-game related streams. We identify three areas for future exploration based on our current work. First, both streamers and improv teams develop fans. Our research did not attempt to build or leverage a fan following. Encouraging long-term engagement between performers and viewers is likely to introduce new design challenges. Second, StAGE currently replicates the theatrical setup of a stage, with the camera located outside the performance space. However, audience perspective in a livestreamed performance could be changed using handheld cameras, virtual representations, and/or editing. These differences would likely enable new types of games and require new performer skills; the language of film could used to communicate more and different performative information. Finally, StAGE does not address the economics of either improv or livestreaming. Improv shows typically require buying a ticket, while Twitch has a strong culture of donation and patronage [18]. How can a system economically support improvisers, without simply becoming a payto-participate model? We look forward to exploring questions like these in the future.

\section{ACKNOWLEDGMENTS}

We would like to thank Joseph Seering for his invaluable insight. We also thank our improvisers Saran Bakari, Jaron Crawford, Ethan Jones-Romero, Arthur Langlie, Amanda Ripley, Emily Suarez, and Jahquan Wynder.

\section{REFERENCES}

[1] AltspaceVR. 2013. AltspaceVR. https://altvr.com/

[2] Rocco Balsamo. 2018. skribbl.io. https://skribbl.io/

[3] Fanni Bányai, Mark D. Griffiths, Orsolya Király, and Zsolt Demetrovics. 2019 The Psychology of Esports: A Systematic Literature Review. fournal of Gambling Studies 35, 2 (01 Jun 2019), 351-365. https://doi.org/10.1007/s10899-018-9763-1

[4] James Batchelor. 2017. Twitch: "We're almost ready to go full-blown Hunger Games". https://www.gamesindustry.biz/articles/2017-11-20-twitch-werealmost-ready-to-go-full-blown-hunger-games

[5] Christopher M. Bingham. 2017. An ethnography of Twitch streamers: negotiating professionalism in new media content creation. Ph.D. Dissertation.

[6] Upright Citizens Brigade. 2018. How to Watch UCB in Virtual Reality. https: //ucbindustries.com/blog/2018/10/3/how-to-watch-ucb-in-virtual-reality

[7] Gifford Cheung and Jeff Huang. 2011. Starcraft from the Stands: Understanding the Game Spectator. In Proceedings of the SIGCHI Conference on Human Factors in Computing Systems (Vancouver, BC, Canada) (CHI '11). Association for Computing Machinery, New York, NY, USA, 763-772. https://doi.org/10.1145/1978942. 1979053

[8] Mary Crossan and Marc Sorrenti. 1997. Making sense of improvisation. Vol. 14 Emerald Publishing, 155-180.
[9] William Cruse, Howard Bay, Clive Barker, and Michael Gillette. 2015. Stagecraft. https://www.britannica.com/art/stagecraft

[10] Jie Deng, Felix Cuadrado, Gareth Tyson, and Steve Uhlig. 2015. Behind the game: Exploring the twitch streaming platform. 2015 International Workshop on Network and Systems Support for Games (NetGames) (2015). https://doi.org/10. 1109/netgames.2015.7382994

[11] Matt Dennie, Alex French, Lou Gonzalez, Yoni Lotan, Caitlin Puckett, and Molly Thomas. 2019. The Instagram Show.

[12] Nicolas Ducheneaut, Robert J. Moore, and Eric Nickell. 2007. Virtual "Third Places": A Case Study of Sociability in Massively Multiplayer Games. Comput. Supported Coop. Work 16, 1-2 (April 2007), 129-166. https://doi.org/10.1007/ s10606-007-9041-8

[13] Dustin Freeman and Ravin Balakrishnan. 2016. Improv Remix: Mixed-Reality Video Manipulation Using Whole-Body Interaction to Extend Improvised Theatre. Proceedings of the 2016 ACM Conference on Designing Interactive Systems - DIS 16 (2016). https://doi.org/10.1145/2901790.2901894

[14] Guo Freeman and Donghee Wohn. 2017. Social Support in eSports: Building Emotional and Esteem Support from Instrumental Support Interactions in a Highly Competitive Environment. 435-447. https://doi.org/10.1145/3116595. 3116635

[15] Daniel Fuller and Brian Magerko. 2010. Shared Mental Models in Improvisational Performance. In Proceedings of the Intelligent Narrative Technologies III Workshop (Monterey, California) (INT3 '10). ACM, New York, NY, USA, Article 15, 6 pages. https://doi.org/10.1145/1822309.1822324

[16] Seth Glickman, Nathan McKenzie, Joseph Seering, Rachel Moeller, and Jessica Hammer. 2018. Design Challenges for Livestreamed Audience Participation Games. In Proceedings of the 2018 Annual Symposium on Computer-Human Interaction in Play (Melbourne, VIC, Australia) (CHI PLAY '18). ACM, New York, NY, USA, 187-199. https://doi.org/10.1145/3242671.3242708

[17] Daniel Gros, Anna Hackenholt, Piotr Zawadzki, and Brigitta Wanner. 2018. Interactions of Twitch Users and Their Usage Behavior. In Social Computing and Social Media. Technologies and Analytics, Gabriele Meiselwitz (Ed.). Springer International Publishing, Cham, 201-213.

[18] Daniel Gros, Brigitta Wanner, Anna Hackenholt, Piotr Zawadzki, and Kathrin Knautz. 2017. World of Streaming. Motivation and Gratification on Twitch. Social Computing and Social Media. Human Behavior Lecture Notes in Computer Science (May 2017), 44-57. https://doi.org/10.1007/978-3-319-58559-8_5

[19] William A. Hamilton, Oliver Garretson, and Andruid Kerne. 2014. Streaming on Twitch: Fostering Participatory Communities of Play Within Live Mixed Media. In Proceedings of the 32Nd Annual ACM Conference on Human Factors in Computing Systems (Toronto, Ontario, Canada) (CHI '14). ACM, New York, NY, USA, 1315-1324. https://doi.org/10.1145/2556288.2557048

[20] Zorah Hilvert-Bruce, James T Neill, Max Sjöblom, and Juho Hamari. 2018. Social motivations of live-streaming viewer engagement on Twitch. Computers in Human Behavior 84 (2018), 58-67.

[21] Mu Hu, Mingli Zhang, and Yu Wang. 2017. Why do audiences choose to keep watching on live video streaming platforms? An explanation of dual identification framework. Computers in Human Behavior 75 (Oct 2017), 594-606. https: //doi.org/10.1016/j.chb.2017.06.006

[22] Discord Inc. 2015. Discord. https://discordapp.com/

[23] Keith Johnstone. 1999. Impro for storytellers. Faber and Faber.

[24] Keith Johnstone. 2019. Impro: improvisation and the theatre. Bloomsbury Academic.

[25] Kat Koppett. 2013. Training to imagine practical improvisational theatre techniques for trainers and managers to enchance creativity, teamwork, leadership, and learning. Stylus.

[26] Peter C. Kunze. 2015. Laughter in the digital age. Comedy Studies 6, 2 (2015), 101-106. https://doi.org/10.10802040610x.2015.1094945

[27] Alex Leavitt. 2014. TwitchPlayedPokemon: An Analysis of the Experimental Interactive Phenomenon. https://gdcvault.com/play/1021438/

[28] Pascal Lessel, Maximilian Altmeyer, and Antonio Krüger. 2018. Viewers' Perception of Elements Used in Game Live-Streams. In Proceedings of the 22Nd International Academic Mindtrek Conference (Tampere, Finland) (Mindtrek '18). ACM, New York, NY, USA, 59-68. https://doi.org/10.1145/3275116.3275117

[29] Pascal Lessel, Michael Mauderer, Christian Wolff, and Antonio Krüger. 2017. Let's Play My Way: Investigating Audience Influence in User-Generated Gaming LiveStreams. In Proceedings of the 2017 ACM International Conference on Interactive Experiences for TV and Online Video (Hilversum, The Netherlands) (TVX '17). ACM, New York, NY, USA, 51-63. https://doi.org/10.1145/3077548.3077556

[30] Pascal Lessel, Alexander Vielhauer, and Antonio Krüger. 2017. Expanding Video Game Live-Streams with Enhanced Communication Channels: A Case Study. In Proceedings of the 2017 CHI Conference on Human Factors in Computing Systems (Denver, Colorado, USA) (CHI '17). ACM, New York, NY, USA, 1571-1576. https: //doi.org/10.1145/3025453.3025708

[31] NightDev LLC. 2017. NightBot. https://docs.nightbot.tv/

[32] Brian Magerko, Waleed Manzoul, Mark Riedl, Allan Baumer, Daniel Fuller, Kurt Luther, and Celia Pearce. 2009. An empirical study of cognition and theatrical 
improvisation. 117-126. https://doi.org/10.1145/1640233.1640253

[33] Brian Magerko and Mark Riedl. 2008. What Happens Next?: Toward an Empirical Investigation of Improvisational Theatre. Proceedings of the International foint Workshop on Computational Creativity 2008 (01 2008).

[34] Claire Mikalauskas, Tiffany Wun, Kevin Ta, Joshua Horacsek, and Lora Oehlberg. 2018. Improvising with an Audience-Controlled Robot Performer. Proceedings of the 2018 on Designing Interactive Systems Conference 2018 - DIS 18 (Jun 2018). https://doi.org/10.1145/3196709.3196757

[35] Keir Miron. 2016. 24 - Seth Sivak of Streamline at E3 2016. http://www.keirmiron com/thequestionbus/24

[36] Moobot. [n.d.]. Moobot. https://moo.bot/

[37] Jȩdrzej Olejniczak. 2015. A Linguistic Study of Language Variety Used on Twitch.Tv: Descriptive and Corpus-Based Approaches. Redefining Community in Intercultural Context 4 (01 2015), 329-334.

[38] Jẹdrzej Olejniczak. 2015. A Linguistic Study of Language Variety Used on Twitch.Tv: Descriptive and Corpus-Based Approaches. Redefining Community in Intercultural Context 4 (01 2015), 329-334.

[39] Dan Patterson and Mark Leveson. 1998-2004. Whose Line Is It Anyway?

[40] Daniel Recktenwald. 2017. Toward a transcription and analysis of live streaming on Twitch. Fournal of Pragmatics 115 (2017), 68-81. https://doi.org/"10.1016/j. pragma.2017.01.013"

[41] Raquel Robinson, Jessica Hammer, and Katherine Isbister. 2019. All the World (Wide Web)'s a Stage: A Workshop on Live Streaming. In Extended Abstracts of the 2019 CHI Conference on Human Factors in Computing Systems (Glasgow, Scotland Uk) (CHI EA '19). ACM, New York, NY, USA, Article W23, 8 pages. https://doi.org/10.1145/3290607.3299016

[42] Michael Rougeau. 2014. Making 'Choice Chamber', A Game For Those Who Like To Be Watched. http://animalnewyork.com/2014/choice-chamber-game-plan/

[43] Jesse Schell. 2019. The Art of Game Design: A Book of Lenses. Taylor \& Francis.

[44] Rainforest Scully-Blaker, Jason Begy, Mia Consalvo, and Sarah Ganzon. 2017. Playing along and Playing for on Twitch: Livestreaming from Tandem Play to Performance. Proceedings of the 50th Hawaii International Conference on System Sciences (2017) (2017). https://doi.org/10.24251/hicss.2017.246

[45] Joseph Seering, Robert Kraut, and Laura Dabbish. 2017. Shaping Pro and AntiSocial Behavior on Twitch Through Moderation and Example-Setting. In Proceedings of the 2017 ACM Conference on Computer Supported Cooperative Work and Social Computing (Portland, Oregon, USA) (CSCW'17). ACM, New York, NY, USA, 111-125. https://doi.org/10.1145/2998181.2998277

[46] Joseph Seering, Michal Luria, Geoff Kaufman, and Jessica Hammer. 2019. Beyond Dyadic Interactions: Considering Chatbots As Community Members. In Proceedings of the 2019 CHI Conference on Human Factors in Computing Systems (Glasgow, Scotland Uk) (CHI '19). ACM, New York, NY, USA, Article 450, 13 pages. https://doi.org/10.1145/3290605.3300680

[47] Joseph Seering, Saiph Savage, Michael Eagle, Joshua Churchin, Rachel Moeller Jeffrey P. Bigham, and Jessica Hammer. 2017. Audience Participation Games:
Blurring the Line Between Player and Spectator. In Proceedings of the 2017 Conference on Designing Interactive Systems (Edinburgh, United Kingdom) (DIS '17). ACM, New York, NY, USA, 429-440. https://doi.org/10.1145/3064663.3064732

[48] Max Sjöblom, Maria Törhönen, Juho Hamari, and Joseph Macey. 2019. The ingredients of Twitch streaming: Affordances of game streams. Computers in Human Behavior 92 (2019), 20 - 28. https://doi.org/10.1016/j.chb.2018.10.012

[49] Thomas Smith, Marianna Obrist, and Peter Wright. 2013. Live-Streaming Changes the (Video) Game. In Proceedings of the 11th European Conference on Interactive TV and Video (Como, Italy) (EuroITV '13). Association for Computing Machinery, New York, NY, USA, 131-138. https://doi.org/10.1145/2465958.2465971

[50] Flavia Sparacino, Kristin Hall, Christopher Wren, and Alex Pentland. 1997. Improvisational Theater Space.

[51] Viola Spolin. 2017. Improvisation for the theater: a handbook of teaching and directing techniques. Martino Fine Books.

[52] T. L. Taylor. 2018. Watch me play twitch and the rise of game live streaming. Princeton University Press.

[53] Charlie Todd and Chelsea Clarke. 2019. The Curfew.

[54] Twitch. 2015. Introducing Twitch Creative. https://blog.twitch.tv/introducingtwitch-creative-fbfe23b4a114

[55] Twitch. 2017. Extensions: A Revolution in Live Streaming. https://www.twitch. tv/p/extensions/

[56] Twitch. 2018. Bring your community together with Rooms. https://blog.twitch. tv/bring-your-community-together-with-rooms-ad60cab1af0a

[57] Twitch. 2019. An Introduction to Twitch Sings. https://help.twitch.tv/s/article/anintroduction-to-twitch-sings

[58] Twitch. 2019. Squad Stream: The next way to play and watch together. https://blog.twitch.tv/squad-stream-the-next-way-to-play-and-watchtogether-32d9ad2ac555

[59] Amy Voida and Saul Greenberg. 2009. Wii All Play: The Console Game As a Computational Meeting Place. In Proceedings of the SIGCHI Conference on Human Factors in Computing Systems (Boston, MA, USA) (CHI '09). ACM, New York, NY, USA, 1559-1568. https://doi.org/10.1145/1518701.1518940

[60] Andrew M. Webb, Chen Wang, Andruid Kerne, and Pablo Cesar. 2016. Distributed Liveness: Understanding How New Technologies Transform Performance Experiences. In Proceedings of the 19th ACM Conference on ComputerSupported Cooperative Work \& Social Computing (San Francisco, California, USA) (CSCW '16). Association for Computing Machinery, New York, NY, USA, 432-437. https://doi.org/10.1145/2818048.2819974

[61] Donghee Yvette Wohn, Guo Freeman, and Caitlin McLaughlin. 2018. Explaining Viewers' Emotional, Instrumental, and Financial Support Provision for Live Streamers. In Proceedings of the 2018 CHI Conference on Human Factors in Computing Systems (Montreal QC, Canada) (CHI '18). ACM, New York, NY, USA, Article 474, 13 pages. https://doi.org/10.1145/3173574.3174048

[62] Cong Zhang and Jiangchuan Liu. 2015. On crowdsourced interactive live streaming. Proceedings of the 25th ACM Workshop on Network and Operating Systems Support for Digital Audio and Video - NOSSDAV 15 (2015). https: //doi.org/10.1145/2736084.2736091 